\title{
Improvement in Patient-Reported Outcomes in Patients with Psoriatic Arthritis Treated with Upadacitinib Versus Placebo or Adalimumab: Results from SELECT-PSA 1
}

\author{
Vibeke Strand · Philip J. Mease - Enrique R. Soriano • Mitsumasa Kishimoto • \\ Carlo Salvarani · Christopher D. Saffore · Patrick Zueger • \\ Erin McDearmon-Blondell · Koji Kato · Dafna D. Gladman
}

Received: August 9, 2021 / Accepted: September 22, 2021 / Published online: October 12, 2021

(C) The Author(s) 2021

\section{ABSTRACT}

Introduction: The aim of this work is to assess the effect of upadacitinib versus adalimumab and placebo on patient-reported outcomes (PROs) in psoriatic arthritis (PsA) patients with inadequate responses to $\geq 1$ non-biologic disease-modifying anti-rheumatic drugs (nonbDMARD-IR) in SELECT PsA-1.

Methods: In this placebo- and active comparator, phase 3 randomized, controlled trial, patients received daily upadacitinib 15 or $30 \mathrm{mg}$, placebo, or adalimumab $40 \mathrm{mg}$ every

Supplementary Information The online version contains supplementary material available at https:// doi.org/10.1007/s40744-021-00379-9.

\section{Strand}

Division of Immunology/Rheumatology, Stanford University, Palo Alto, CA, USA

P. J. Mease

Department of Rheumatology, Swedish Medical Center, Providence St Joseph Health and University of Washington, Seattle, WA, USA

\section{E. R. Soriano}

Department of Public Health, Rheumatology Unit, Internal Medicine Services, Hospital Italiano de Buenos Aires, Instituto Universitario Hospital Italiano de Buenos Aires, Buenos Aires, Argentina

M. Kishimoto

Department of Nephrology and Rheumatology, Kyorin University School of Medicine, Tokyo, Japan other week through 56 weeks. At week 24, placebo-assigned patients were rerandomized to upadacitinib 15 or $30 \mathrm{mg}$. PROs included Patient Global Assessment of Disease Activity (PtGA), pain, Health Assessment Questionnaire Disability Index (HAQ-DI), Functional Assessment of Chronic Illness Therapy-Fatigue (FACIT-F), Short Form 36 Health Survey (SF-36), EQ-5D-5L index score, Bath Ankylosing Spondylitis Disease Activity Index, morning stiffness, Self-Assessment of Psoriasis Symptoms, and Work Productivity and Activity Impairment. Mean changes from baseline in PROs, improvements $\geq$ minimum clinically important differences (MCID), scores $\geq$ normative values, and sustained clinically meaningful responses were compared between treatment groups.

C. Salvarani

Rheumatology Units, University of Modena and Reggio Emilia, Azienda USL-IRCCS di Reggio Emilia, Reggio Emilia, Italy

C. D. Saffore $(\bowtie) \cdot$ P. Zueger .

E. McDearmon-Blondell · K. Kato

Health Economics and Outcomes Research, AbbVie

Inc., 1 N Waukegan Road, Dept. ABV1-4NW-105-03,

North Chicago, IL 60064-6078, USA

e-mail: christopher.saffore@abbvie.com

D. D. Gladman

Department of Medicine, Schroeder Arthritis Institute, Krembil Research Institute, Toronto Western Hospital, University of Toronto, Toronto, ON, Canada 
Results: At weeks 12 and 24, upadacitinib treatment resulted in improvements from baseline versus placebo across all PROs as well as improvements versus adalimumab in HAQ-DI and SF-36 Physical Component Summary score (nominal $p<0.05$ ). Improvements in PtGA, pain, and HAQ-DI were reported as early as week 2. At week 12, significantly (nominal $p<0.05)$ more upadacitinib- versus placebotreated patients reported improvements $\geq$ MCID across all PROs including seven SF-36 domains. The proportions of upadacitinib-treated patients reporting clinically meaningful improvements at week 12 were similar to or greater than with adalimumab and sustained through week 56. Significantly (nominal $p<0.05$ ) more upadacitinib-treated (both doses) patients reported scores $\geq$ normative values at week 12 versus placebo, and scores were generally similar to or greater than adalimumab.

Conclusions: Upadacitinib treatment provides rapid, sustained, and clinically meaningful improvements in PROs in non-bDMARD-IR patients with PsA.SELECT-PsA 1 ClinicalTrials.gov number, NCT03104400.

Keywords: Adalimumab; Disease-modifying anti-rheumatic drugs; Pain; Patient-reported outcomes; Physical function; Psoriatic arthritis; Quality of life; Work productivity; Upadacitinib

\section{Key Summary Points}

Why carry out this study?

Psoriatic arthritis is a multifaceted disease with substantial negative impact on health-related quality of life.

Despite various current treatment options, not all patients achieve disease control, demonstrating a need for new treatment options.
This trial provides a head-to-head comparison of patient-reported outcomes in patients treated with upadacitinib, an oral, Janus kinase inhibitor and adalimumab, a well-characterized tumor necrosis factor inhibitor commonly used to treat moderate and severe psoriatic arthritis.

\section{What was learned from the study?}

In patients with psoriatic arthritis and inadequate responses to non-biologic disease-modifying anti-rheumatic drugs, treatment with upadacitinib for 12 weeks resulted in clinically meaningful improvements in patient-reported outcomes that were maintained or further improved at weeks 24 and 56.

Overall, improvements with upadacitinib were similar to or greater than those reported with adalimumab.

\section{INTRODUCTION}

Psoriatic arthritis (PsA) is a heterogenous, inflammatory musculoskeletal disease characterized by peripheral arthritis, dactylitis, enthesitis, and axial arthritis, as well as skin and nail disease [1]. PsA is associated with significant psychosocial burden that negatively impacts work productivity, health-related quality of life (HRQoL), [2] and activities of daily living [3].

Treatment goals are to alleviate disease symptoms, prevent structural damage, and maximize HRQoL. Non-steroidal anti-inflammatory drugs (NSAIDs), corticosteroids, and non-biologic disease-modifying anti-rheumatic drugs (non-bDMARDs) are often used to treat mild disease [4-6]. bDMARDs and targeted synthetic DMARDs (tsDMARDs) are recommended in patients with severe disease or refractory disease and tumor necrosis factor (TNF) inhibitors are often used as first-line therapy [4-6]. In patients with inadequate responses to non-bDMARDs (non-bDMARD-IR) or TNF inhibitors (TNFi), other treatment options include interleukin (IL)-12/23, IL-17A, 
and recently, IL-23 inhibitors, and tsDMARDs: Janus kinase inhibitors [4-6].

Upadacitinib is an oral reversible Janus kinase inhibitor [7] with demonstrated efficacy in the treatment of rheumatoid arthritis [8-12]. Adalimumab is a TNFi indicated for treatment of multiple diseases, including PsA $[5,6,13]$. Head-to-head comparisons of the effects of upadacitinib versus adalimumab on the multiple clinical aspects of PsA provide physicians with important information to guide their decisions regarding the treatment of this disease.

SELECT-PsA 1 was a phase 3 randomized controlled trial (RCT) comparing upadacitinib $15 \mathrm{mg}$ once daily (QD), upadacitinib $30 \mathrm{mg}$ QD, placebo QD, or adalimumab $40 \mathrm{mg}$ every other week (EOW) in non-bDMARD-IR PsA patients. Key findings from the SELECT-PsA 1 study included significant improvements in the clinical manifestations of PsA, such as musculoskeletal outcomes, psoriasis severity, axial symptoms, as well as resolution of dactylitis and enthesitis [14]. Since PsA significantly impacts HRQoL, it is important to assess the effect of upadacitinib on patient-reported outcomes (PROs) when evaluating treatment benefits in PsA [15]. This report presents an evaluation of the attainment and maintenance of clinically meaningful improvements in PROs in PsA patients receiving upadacitinib versus placebo or adalimumab in the SELECT-PsA 1 trial.

\section{METHODS}

\section{Study Design and Patients}

The design and primary results from the SELECT-PsA 1 RCT have previously been reported [14]. The RCT was conducted at 281 sites in 45 countries. Key inclusion criteria in SELECTPsA 1 were patients $\geq 18$ years of age, fulfillment of the ClASsification criteria for Psoriatic ARthritis (CASPAR) [16], active or documented history of plaque psoriasis, and inadequate responses to $\geq 1$ non-bDMARD. Major exclusion criteria were prior exposure to any bDMARDs, prior exposure to any JAK inhibitor, current treatment with $\geq 2$ non-bDMARDs, current or past history of infection, and underlying medical diseases or problems.

Patients were randomly assigned in a 1:1:1:1 ratio to upadacitinib $15 \mathrm{mg}$ QD, upadacitinib $30 \mathrm{mg}$ QD, placebo followed by upadacitinib $15 \mathrm{mg}$ QD or $30 \mathrm{mg}$ QD (1:1) at week 24, or adalimumab $40 \mathrm{mg}$ EOW. Study drug (upadacitinib, adalimumab, and placebo) was provided by AbbVie. The trial consisted of 24 weeks of randomized, blinded, placebo- and active comparator-controlled treatment followed by 32 weeks of active-comparator-controlled treatment. Stable treatment with nonsteroidal antiinflammatory drugs (NSAID), corticosteroids, and $\leq 2$ non-bDMARDs was permitted, but not required. Rescue therapy was permitted for nonresponders at week 16.

The SELECT-PSA 1 RCT was conducted according to the International Conference on Harmonization guidelines and the principles of the Declaration of Helsinki. All patients provided written informed consent. The trial protocol was approved by independent ethics committees and institutional review boards (ESM Table S1).

\section{Outcomes}

Multiplicity-controlled secondary PRO endpoints included changes from baseline at week 12 in Health Assessment Questionnaire Disability Index (HAQ-DI; minimum clinically important difference [MCID]: $\geq 0.35$ unit decrease; normative value: $\leq 0.25$ units) $[17,18]$, Functional Assessment of Chronic Illness Therapy-Fatigue (FACIT-F; MCID: $\geq 4$ point increase; normative value: $\geq 40.1$ points) $[19,20]$, Short Form 36 Health Survey (SF-36) Physical Component Summary (PCS) score (MCID: $\geq 2.5$ increase; normative value $\geq 50$ points) [21-23] and changes from baseline at week 16 in Self-Assessment of Psoriasis Symptoms (SAPS) (an 11-item patient self-assessment of psoriasis symptoms, which included severity of pain, itching, redness, scaling, flaking, bleeding, burning, stinging, tenderness, pain due to skin cracking, and joint pain in the areas affected by psoriasis; range $0-110$, with higher scores indicating worse patient-reported 
psoriasis symptoms) [24]. Additional PROs evaluated included Patient Global Assessment of Disease Activity (PtGA) and pain (0-10 NRS; MCID: $\geq 1$-point decrease; normative value: $\leq 2$ points; pain was a multiplicity-controlled secondary PRO) $[23,25,26]$, SF-36 Mental Component Summary (MCS; MCID: $\geq 2.5$ point increase; normative value: $\geq 50$ points) and individual SF-36 domain scores (MCID: $\geq$ five-point increase) [21-23], EuroQoL 5-Dimension 5-Level index score (EQ-5D-5L; MCID: $\geq 0.05$-unit increase; normative value: $\geq 0.915)$ [27, 28], Bath Ankylosing Spondylitis Disease Activity Index (BASDAI; MCID: $\geq 1.1$ point decrease), morning stiffness (mean of BASDAI questions 5 and 6) [29], BASDAI 50, Work Productivity and Activity Impairment (WPAI) [30, 31], and itch (SAPS question 2; 0-10 NRS, with higher scores indicating worse itch).

PtGA, pain, and HAQ-DI were assessed starting at week 2 while other PROs were assessed at week 12, except SAPS at week 16. SF-36 PCS and MCS are norm-based with a mean value of 50 and standard deviation of 10; SF-36 domains were scored from 0 to 100 with higher scores indicating better HRQoL [21, 22]. Ageand gender-matched US normative SF-36 domain scores were based on the protocol population [32]. BASDAI was assessed in patients who had investigator-determined psoriatic spondylitis at baseline. The WPAI activity impairment domain was evaluated in all patients while presenteeism, overall work impairment, and absenteeism domains were assessed in those employed at baseline [31].

\section{Statistical Analyses}

All analyses were conducted on the full analysis set of randomized patients who received $\geq 1$ dose of trial drug. As previously described [14], the RCT was powered to detect a difference between placebo and upadacitinib for the primary endpoint (ACR 20 response), most key secondary endpoints, and evaluating noninferiority and superiority of each upadacitinib dose compared with adalimumab for ACR20 response at week 12 . The study was not powered to detect differences between the upadacitinib groups nor to detect differences between upadacitinib and adalimumab with respect to the change from baseline in pain or HAQ-DI score. Demographic and baseline characteristics are summarized with descriptive statistics (mean, standard deviation for continuous endpoints, and $n[\%]$ for categoric endpoints). Least squares (LS) mean changes from baseline through weeks 12,24 , and 56 along with $95 \%$ confidence intervals (CIs) and nominal $p$ values were based on mixed-effects repeated measures models (MMRM) analysis using an unstructured variance-covariance matrix, including treatment, visit, treatment-by-visit interaction, the stratification factor, current non-bDMARD use (yes/no) as fixed factors and the continuous fixed covariate of baseline measurement. MMRM analysis used observed longitudinal data up to the respective time point prior to premature study drug discontinuation. Spidergrams were used to illustrate changes from baseline in SF-36 domain scores in each treatment group against a combined baseline US normative population [32]. No formal comparisons of adalimumab versus placebo were performed.

The proportions of patients reporting BASDAI 50, improvements $\geq$ MCID, and $\geq$ normative values (age- and gender-matched for SF-36 domains) at weeks 12 and 24 were evaluated between both doses of upadacitinib and placebo or between both doses of upadacitinib and adalimumab using nonresponder imputation for missing responses. As-observed data were used to determine maintenance of clinically meaningful improvements $\geq$ MCID from weeks 12 to 56 . The proportions of patients reporting scores $\geq$ normative values in respective PROs including SF-36 domains through week 24 were determined with missing responses imputed using nonresponder imputation.

The number needed to treat (NNT) to achieve one additional MCID improvement at weeks 12 and 24 for each upadacitinib dose compared with placebo or adalimumab $40 \mathrm{mg}$ was defined as the reciprocal of the response rate difference between upadacitinib and placebo or adalimumab, with missing PRO responses imputed using nonresponder imputation. $P$ values were calculated using 
Cochran-Mantel-Haenszel test adjusting for the main stratification factor of current nonbDMARD use (yes/no). Statistical significance defined as $p<0.05$ was nominal for non-multiplicity-controlled endpoints.

\section{RESULTS}

A total of 1705 patients in SELECT-PsA 1 were randomized with 1704 receiving $\geq 1$ dose of study drug (placebo: $n=423$; upadacitinib $15 \mathrm{mg}: n=429$; upadacitinib $30 \mathrm{mg}: n=423$; adalimumab $40 \mathrm{mg}: \quad n=429$ ) with approximately $58 \%$ of the patients employed. Demographics, disease characteristics, disease activity, and PRO scores at baseline were similar across the treatment groups (Table 1).

\section{Improvements from Baseline in PROs}

Significant improvements in LS mean changes from baseline to week 12 and 24 were reported with upadacitinib $15 \mathrm{mg}$ and upadacitinib $30 \mathrm{mg}$ compared with placebo across all PROs (Table 2). Improvements from baseline in PtGA, pain, HAQ-DI, and FACIT-F with both doses of upadacitinib differentiated from placebo as early as 2 weeks (Fig. 1).

As shown in Fig. 2, decrements from age- and gender-adjusted norms indicated that patients had substantial impairments in HRQoL at baseline. All eight SF-36 domain scores were significantly improved with upadacitinib versus placebo with scores approaching normative values at week 12 (Fig. 2). Improvements in PROs reported at week 12 with upadacitinib continued or further increased at week 24 (Table 2; ESM Fig. S1). BASDAI 50 response was reported by significantly more patients with upadacitinib $15 \mathrm{mg}$ or $30 \mathrm{mg}$ at weeks 12 (37\% and 51\%) and $24(59 \%$ and 53\%) than placebo $(15 \%$ and $27 \%)$ (nominal $p<0.001$ ).

Compared with adalimumab, both upadacitinib doses resulted in significant improvements in HAQ-DI and SF-36 PCS scores, and SF-36 physical function (PF) domain with upadacitinib $15 \mathrm{mg}$ and $\mathrm{PF}$, role physical (RP), bodily pain (BP), and general health (GH) domains with upadacitinib $30 \mathrm{mg}$ at weeks 12 and 24; and in SAPS and itch response at weeks 16 and 24 (Table 2). Improvements in PROs with both doses of upadacitinib were maintained or further improved at 56 weeks of treatment (Table 3).

Patients receiving placebo who switched to upadacitinib $15 \mathrm{mg}$ or upadacitinib $30 \mathrm{mg}$ at week 24 reported improvements in PtGA, pain, HAQ-DI, and FACIT-F similar to those who initially received either upadacitinib dose. These improvements were maintained through week 56 (Fig. 1).

LS mean changes in SAPS score were significantly greater in patients treated with both doses of upadacitinib compared with placebo and indicative of important improvements in the signs and symptoms of psoriasis (Table 2). The itch response rate (derived from SAPS question 2) was greater in both upadacitinib groups compared with placebo at week 16 (33.8\% for upadacitinib $15 \mathrm{mg}, 48.3 \%$ for upadacitinib $30 \mathrm{mg}$ versus $9.6-13.5 \%$ for placebo; ESM, Fig. S2). With upadacitinib $15 \mathrm{mg}$ and $30 \mathrm{mg}$, itch responses improved to $45.5 \%$ and $54.3 \%$ at week 56 , respectively. In patients who switched from placebo to upadacitinib at week 24, itch responses approached those reported by patients who initially received upadacitinib.

\section{Clinically Meaningful Improvements in PROs}

As early as week 2, significantly (nominal $p<0.01)$ more patients treated with upadacitinib $15 \mathrm{mg}$ and $30 \mathrm{mg}$ reported clinically meaningful improvements $\geq$ MCID in PtGA (15 mg: 67.8\%, $30 \mathrm{mg}: 73.5 \%$ versus placebo: $54.1 \%$ ), pain (15 mg: $64.2 \%$, $30 \mathrm{mg}: 74.6 \% \%$ versus placebo: $51.7 \%$ ), and HAQ-DI (15 mg: $31.0 \%$, $30 \mathrm{mg}: 37.4 \%$ versus placebo: $21.7 \%$ ) compared with placebo.

At week 12, the proportions of patients reporting improvements $\geq$ MCID were significantly greater with upadacitinib $15 \mathrm{mg}$ and $30 \mathrm{mg}$ compared with placebo in PtGA, pain HAQ-DI, FACIT-F, EQ-5D-5L, BASDAI, morning stiffness, and SF-36 PCS scores, as well as seven of eight SF-36 domains (Fig. 3) and maintained 
Table 1 Baseline characteristics and PRO scores

\begin{tabular}{|c|c|c|c|c|}
\hline & $\begin{array}{l}\text { Placebo } \\
(N=423)\end{array}$ & $\begin{array}{l}\text { Upadacitinib } 15 \mathrm{mg} \\
\text { QD }(N=429)\end{array}$ & $\begin{array}{l}\text { Upadacitinib } 30 \mathrm{mg} \\
\text { QD }(N=423)\end{array}$ & $\begin{array}{l}\text { Adalimumab } 40 \mathrm{mg} \\
\text { EOW }(N=429)\end{array}$ \\
\hline Female, \% & 49.9 & 55.5 & 55.8 & 51.7 \\
\hline Age (years), mean (SD) & $50.4(12.2)$ & $51.6(12.2)$ & $49.9(12.4)$ & $51.4(12.0)$ \\
\hline White race, \% & 89.1 & 90.0 & 89.1 & 87.4 \\
\hline $\begin{array}{l}\text { Duration since PsA diagnosis } \\
\text { (years), mean (SD) }\end{array}$ & $6.2(7.0)$ & $6.2(7.4)$ & $5.9(6.4)$ & $5.9(7.1)$ \\
\hline $\begin{array}{l}\text { Body mass index }\left(\mathrm{kg} / \mathrm{m}^{2}\right) \text {, } \\
\text { mean }\end{array}$ & $30.4(6.8)$ & $30.1(6.4)$ & $30.1(6.8)$ & $30.7(7.2)$ \\
\hline $\begin{array}{l}\text { Use of } \geq 1 \text { non-bDMARD } \\
\text { at baseline, } \%\end{array}$ & 82.0 & 82.3 & 81.8 & 80.9 \\
\hline Presence of dactylitis, ${ }^{\mathrm{a}} \%$ & 29.8 & 31.7 & 30.0 & 29.6 \\
\hline Presence of enthesitis, ${ }^{\mathrm{b}} \%$ & 57.0 & 62.9 & 63.1 & 61.8 \\
\hline TJC (68 joints), mean (SD) & $20.0(14.3)$ & $20.4(14.7)$ & $19.4(13.3)$ & $20.1(13.8)$ \\
\hline SJC (66 joints), mean & $11.0(8.2)$ & $11.6(9.3)$ & $10.6(7.1)$ & $11.6(8.8)$ \\
\hline $\mathrm{BSA}-\mathrm{PsO} \geq 3 \%, \%$ & 49.9 & 49.9 & 49.6 & 49.2 \\
\hline PtGA $0-10$, NRS, mean (SD) & $6.3(2.0)$ & $6.6(2.0)$ & $6.4(2.1)$ & $6.3(2.0)$ \\
\hline Pain $0-10$, NRS, mean (SD) & $6.1(2.1)$ & $6.2(2.1)$ & $5.9(2.1)$ & $6.0(2.1)$ \\
\hline HAQ-DI, mean (SD) & $1.12(0.64)$ & $1.15(0.65)$ & $1.09(0.63)$ & $1.12(0.63)$ \\
\hline FACIT-F, mean (SD) & $30.0(11.2)$ & $29.0(11.9)$ & $29.8(11.6)$ & $29.5(11.5)$ \\
\hline SF-36 PCS, mean (SD) & $35.1(8.4)$ & $34.8(7.7)$ & $35.8(8.2)$ & $35.8(8.1)$ \\
\hline SF-36 MCS, mean (SD) & $45.6(11.3)$ & $44.7(11.7)$ & $45.6(11.5)$ & $45.0(11.0)$ \\
\hline \multicolumn{5}{|l|}{ SF-36 domains, mean (SD) } \\
\hline $\mathrm{PF}$ & $42.7(25.6)$ & $43.7(24.3)$ & $46.0(25.2)$ & $45.2(25.5)$ \\
\hline $\mathrm{RP}$ & $42.6(24.0)$ & $41.2(22.6)$ & $46.4(24.0)$ & $44.2(24.7)$ \\
\hline $\mathrm{BP}$ & $36.3(17.6)$ & $34.8(17.0)$ & $36.8(17.6)$ & $37.1(17.8)$ \\
\hline $\mathrm{GH}$ & $42.5(19.1)$ & $41.2(18.3)$ & $41.4(18.6)$ & $41.8(17.3)$ \\
\hline VT & $42.4(20.0)$ & $40.2(20.5)$ & $42.3(20.7)$ & $41.9(19.6)$ \\
\hline SF & $60.3(25.0)$ & $57.3(26.3)$ & $60.2(26.4)$ & $60.6(24.9)$ \\
\hline $\mathrm{RE}$ & $64.8(27.9)$ & $64.4(26.6)$ & $66.5(26.6)$ & $63.9(27.8)$ \\
\hline $\mathrm{MH}$ & $60.5(19.2)$ & $59.9(20.7)$ & $61.3(20.8)$ & $60.1(20.2)$ \\
\hline EQ-5D-5L, mean (SD) & $0.61(0.24)$ & $0.60(0.25)$ & $0.61(0.24)$ & $0.62(0.25)$ \\
\hline BASDAI, ${ }^{\mathrm{c}}$ mean $(\mathrm{SD})$ & $5.4(2.1)$ & $5.6(2.2)$ & $5.4(2.2)$ & $5.4(2.2)$ \\
\hline $\begin{array}{l}\text { Morning stiffness, }{ }^{\mathrm{c}, \mathrm{d}} \text { mean } \\
\quad(\mathrm{SD})\end{array}$ & $5.3(2.5)$ & $5.7(2.6)$ & $5.3(2.6)$ & $5.2(2.6)$ \\
\hline
\end{tabular}


Table 1 continued

\begin{tabular}{|c|c|c|c|c|}
\hline & $\begin{array}{l}\text { Placebo } \\
(N=423)\end{array}$ & $\begin{array}{l}\text { Upadacitinib } 15 \mathrm{mg} \\
\text { QD }(N=429)\end{array}$ & $\begin{array}{l}\text { Upadacitinib } 30 \mathrm{mg} \\
\text { QD }(N=423)\end{array}$ & $\begin{array}{l}\text { Adalimumab } 40 \mathrm{mg} \\
\text { EOW }(N=429)\end{array}$ \\
\hline SAPS, mean (SD) & $44.0(26.6)$ & $44.3(26.9)$ & $43.2(26.2)$ & $42.7(25.3)$ \\
\hline Itch, ${ }^{e}$ mean $(S D)$ & $4.6(3.1)$ & $4.5(3.1)$ & $4.5(3.1)$ & $4.5(3.0)$ \\
\hline WPAI AI, mean (SD) & $49.6(25.0)$ & $52.0(25.2)$ & $46.5(26.2)$ & $49.3(25.9)$ \\
\hline $\begin{array}{l}\text { WPAI presenteeism, }{ }^{\mathrm{f}} \text { mean } \\
(\mathrm{SD})\end{array}$ & $43.6(24.8)$ & $43.0(25.6)$ & $38.3(26.1)$ & $38.3(24.4)$ \\
\hline WPAI OWI, ${ }^{\mathrm{f}}$ mean $(\mathrm{SD})$ & $50.8(29.0)$ & $48.3(29.0)$ & $44.8(30.2)$ & $44.8(28.8)$ \\
\hline $\begin{array}{l}\text { WPAI absenteeism, }{ }^{\mathrm{f}} \text { mean } \\
\text { (SD) }\end{array}$ & $16.3(28.2)$ & $11.7(24.5)$ & $12.9(25.4)$ & $12.8(26.4)$ \\
\hline
\end{tabular}

$A I$ activity impairment, $B A S D A I$ Bath Ankylosing Spondylitis Disease Activity Index, $B P$ bodily pain, $B S A$ body surface area, $b D M A R D$ biologic disease-modifying anti-rheumatic drug, $E O W$ every other week, $E Q-5 D-5 L$ EuroQoL 5-Dimension 5-Level index score, FACIT-F Functional Assessment of Chronic Illness Therapy-Fatigue, GH general health, HAQ-DI Health Assessment Questionnaire Disability Index, MCS Mental Component Summary, $M H$ mental health, NRS numerical rating scale, $O W I$ overall work impairment, $P C S$ Physical Component Summary, $P F$ physical functioning, $P s O$ psoriasis, $P s A$ psoriatic arthritis, $P t G A$ Patient Global Assessment of Disease Activity, $Q D$ once daily, $R E$ role emotional, $R P$ role physical, SAPS Self-Assessment of Psoriasis Symptoms, $S D$ standard deviation, $S F$ social functioning, $S F$-36 36-Item Short Form Health Survey, SJC swollen joint count, TJC tender joint count, $V T$ vitality, WPAI Work Productivity and Activity Impairment

${ }^{a}$ Defined as Leeds Dactylitis Index $>0$

${ }^{b}$ Defined as Leeds Enthesitis Index $>0$

c Reported only for patients with investigator-determined psoriatic spondylitis at baseline

d Mean of BASDAI questions 5 and 6

e SAPS question 2

${ }^{\mathrm{f}}$ Reported only for patients who were employed. $\mathrm{N}$ for presenteeism, OWI, and absenteeism: placebo: 230, 241, and 241; upadacitinib $15 \mathrm{mg}$ QD: 240, 251, and 251; upadacitinib $30 \mathrm{mg}$ QD: 242, 251, and 251; adalimumab $40 \mathrm{mg}$ EOW: 230, 243 , and 243

or further improved at week 24 (ESM Fig. S3). At week 12, the proportions of upadacitinib-treated patients reporting clinically meaningful improvements were similar or greater than with adalimumab $40 \mathrm{mg}$, with exception of SF-36 role emotional (RE) domain. Of note, significantly more patients treated with upadacitinib $30 \mathrm{mg}$ versus adalimumab $40 \mathrm{mg}$ reported clinically meaningful improvements in pain as early as week $2(74.6 \%$ versus $67.1 \%$, nominal $p=0.017)$; sustained to week 56 . Clinically meaningful improvements reported at week 12 continued or were further improved at week 56 with upadacitinib and adalimumab across all PROs (ESM Fig. S4).
At baseline, less than $11 \%$ of patients reported scores within the normative range in PtGA, EQ-5D-5L, SF-36 PCS, and four SF-36 domain scores (Fig. 4). At week 12, significantly (nominal $p<0.05$ ) more patients receiving both doses of upadacitinib reported scores $\geq$ normative values in PtGA, HAQ-DI, FACIT-F, EQ-5D-5L, SF-36 PCS, and matched normative values in seven SF-36 domains compared with placebo (Fig. 4); scores were maintained or improved to week 24 (ESM Fig. S5) and were generally similar to or greater than with adalimumab (Fig. 4 and ESM Fig. S5).

NNTs for upadacitinib $15 \mathrm{mg}$ and upadacitinib $30 \mathrm{mg}$ ranged from 3.0 to 13.2 across all PROs at week 12 with NNTs $\leq 10$ considered 


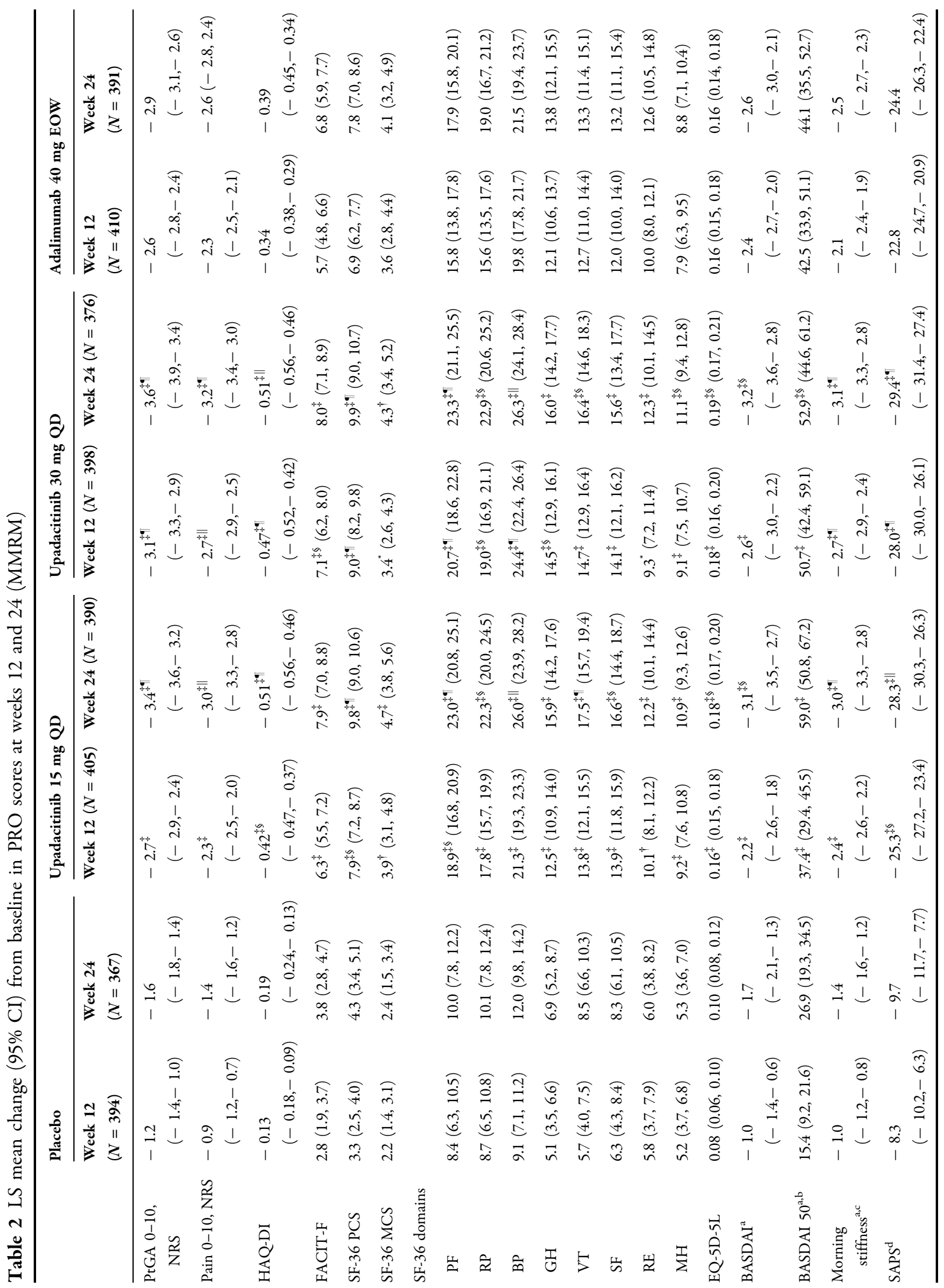




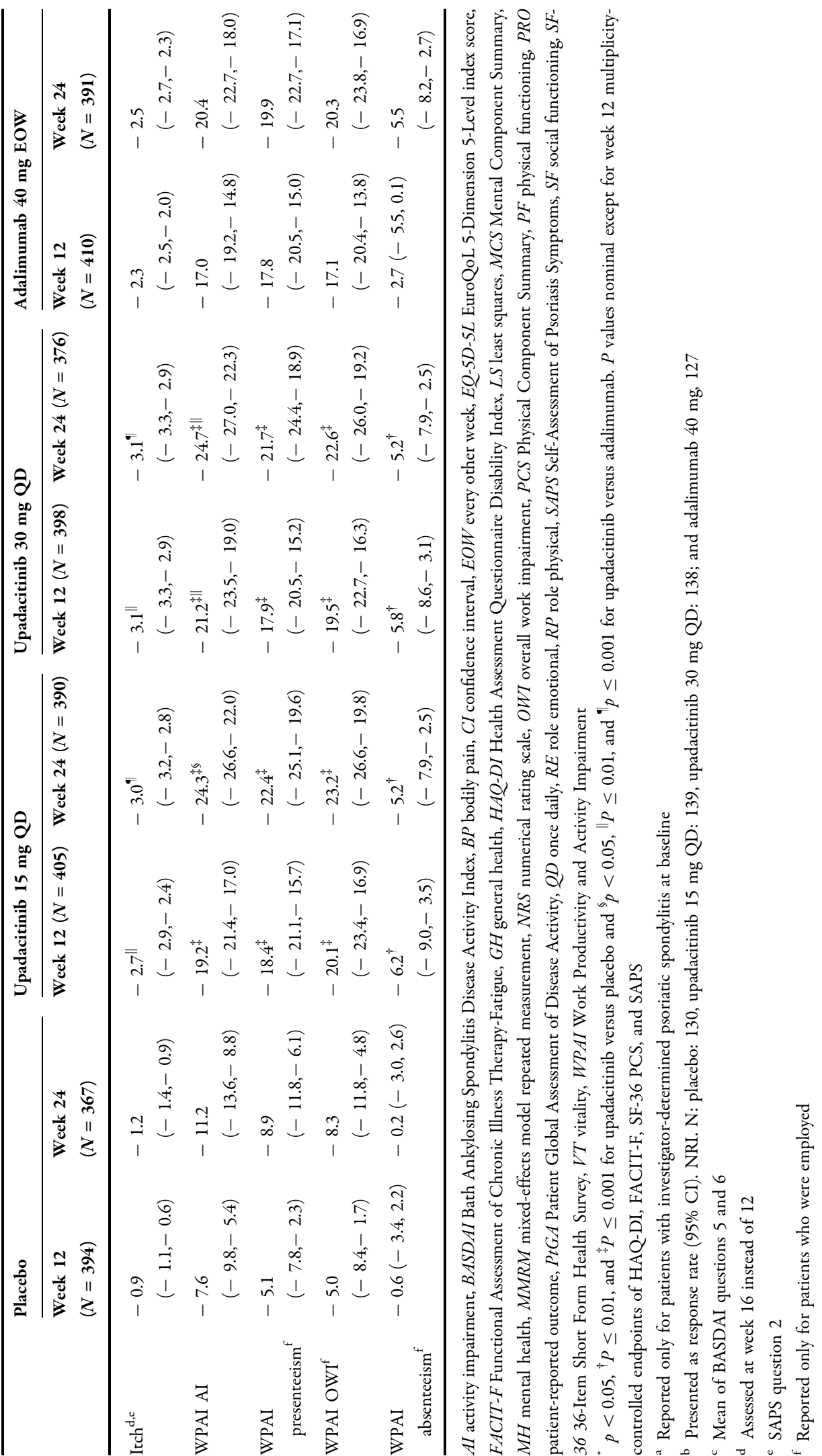



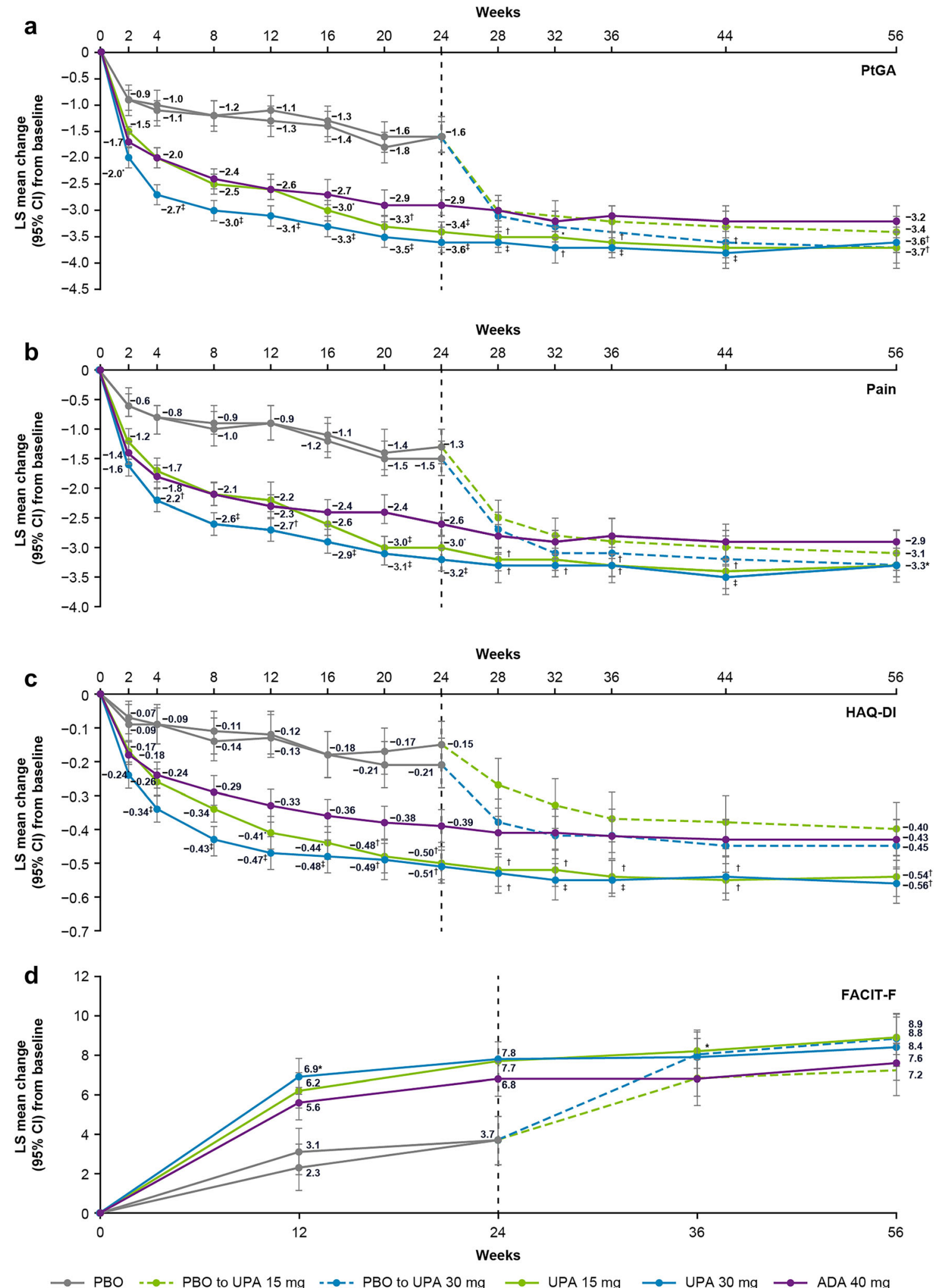
4Fig. 1 Change from baseline through Week 56 in PtGA, pain, HAQ-DI, and FACIT-F over time (MMRM). a PtGA, b Pain, c HAQ-DI, d FACIT-F. ${ }^{*} p<0.05$, $p \leq 0.01$, and ${ }^{*} p \leq 0.001$ for UPA versus ADA. ADA adalimumab, CI confidence interval, FACIT-F Functional Assessment of Chronic Illness Therapy-Fatigue, HAQ-DI Health Assessment Questionnaire Disability Index, LS least squares, MMRM mixed-effects model repeated measurement, PBO placebo, PtGA Patient Global Assessment of Disease Activity, UPA upadacitinib

clinically meaningful (Fig. 3). The range of NNTs with upadacitinib $15 \mathrm{mg}$ and $30 \mathrm{mg}$ versus placebo was generally lower than for adalimumab $40 \mathrm{mg}$ versus placebo at both 12 and 24 weeks (Fig. 3 and ESM Fig. S3).

\section{DISCUSSION}

The SELECT-PsA 1 RCT compared upadacitinib 15 and $30 \mathrm{mg}$ with placebo and adalimumab as an active comparator in non-bDMARD-IR patients with PsA [14]. In this post hoc analysis of the SELECT-PsA 1 trial, patients receiving upadacitinib 15 and $30 \mathrm{mg}$ reported significant and clinically meaningful improvements across a broad variety of PROs evaluating disease activity, pain, physical function, fatigue, psoriasis symptom severity, HRQoL, and work productivity at weeks 12 and 24 compared with placebo. Significant improvements in PtGA, pain, and HAQ-DI with upadacitinib were reported as early as week 2 suggesting rapid

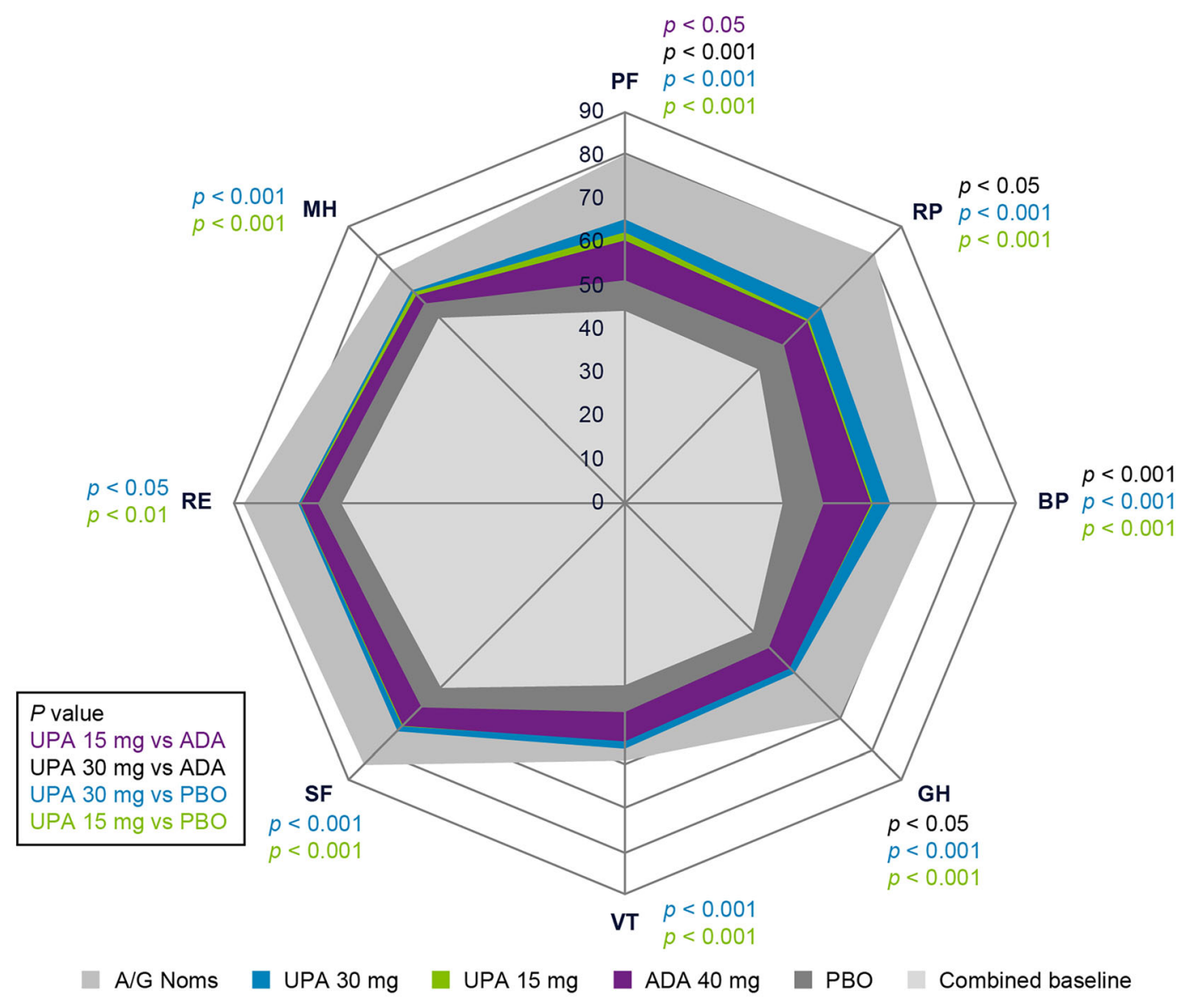

Fig. 2 SF-36 domain scores at Week 12 relative to age- and gender-adjusted normative values (MMRM). $A D A$ adalimumab, $A / G$ norms age- and gender-matched normative values, $B L$ baseline, $B P$ bodily pain, $G H$ general health, $M H$ mental health, $M M R M$ mixed-effects model

repeated measurement, $P B O$ placebo, $P F$ physical functioning, $R E$ role emotional, $R P$ role physical, $S F$ social functioning, $S F$-36 36-Item Short Form Health Survey, $U P A$ upadacitinib, US United States, $V T$ vitality 
Table 3 LS mean change (95\% CI) from baseline in PRO scores at week 56 (MMRM)

\begin{tabular}{|c|c|c|c|}
\hline & $\begin{array}{l}\text { Upadacitinib } 15 \mathrm{mg} \text { QD } \\
(N=371)\end{array}$ & $\begin{array}{l}\text { Upadacitinib } 30 \mathrm{mg} \text { QD } \\
(N=362)\end{array}$ & $\begin{array}{l}\text { Adalimumab } 40 \mathrm{mg} \text { EOW } \\
(N=364)\end{array}$ \\
\hline PtGA $0-10$, NRS & $-3.7(-3.9,-3.4)^{\dagger}$ & $-3.6(-3.9,-3.4)^{\dagger}$ & $-3.2(-3.4,-2.9)$ \\
\hline Pain $0-10$, NRS & $-3.3(-3.6,-3.1)^{*}$ & $-3.3(-3.6,-3.1)^{*}$ & $-2.9(-3.1,-2.7)$ \\
\hline HAQ-DI & $-0.54(-0.59,-0.48)^{\dagger}$ & $-0.56(-0.61,-0.50)^{\dagger}$ & $-0.43(-0.49,-0.38)$ \\
\hline FACIT-F & $8.9(8.0,9.9)$ & $8.4(7.4,9.4)$ & $7.6(6.7,8.6)$ \\
\hline SF-36 PCS & $10.8(10.0,11.7)^{\dagger}$ & $10.5(9.6,11.4)^{\dagger}$ & $8.9(8.0,9.8)$ \\
\hline SF-36 MCS & $5.2(4.2,6.1)$ & $4.4(3.4,5.3)$ & $4.3(3.4,5.2)$ \\
\hline \multicolumn{4}{|l|}{ SF-36 domains } \\
\hline $\mathrm{PF}$ & $25.6(23.2,27.9)^{\dagger}$ & $24.9(22.5,27.2)^{\dagger}$ & $20.5(18.2,22.8)$ \\
\hline $\mathrm{RP}$ & $25.2(22.9,27.5)^{*}$ & $24.6(22.3,27.0)$ & $21.6(19.3,23.9)$ \\
\hline $\mathrm{BP}$ & $28.6(26.2,30.9)^{\dagger}$ & $28.8(26.4,31.2)^{\dagger}$ & $24.1(21.7,26.4)$ \\
\hline $\mathrm{GH}$ & $17.4(15.6,19.3)$ & $15.5(13.6,17.3)$ & $15.3(13.5,17.2)$ \\
\hline VT & $19.9(17.9,21.9)^{*}$ & $16.4(14.4,18.4)$ & $15.0(13.0,17.0)$ \\
\hline SF & $17.9(15.6,20.1)$ & $17.7(15.5,19.9)$ & $14.9(12.7,17.2)$ \\
\hline $\mathrm{RE}$ & $14.2(12.0,16.4)$ & $13.1(10.9,15.3)$ & $13.4(11.3,15.6)$ \\
\hline $\mathrm{MH}$ & $12.1(10.4,13.8)^{*}$ & $11.1(9.4,12.8)$ & $9.6(7.9,11.4)$ \\
\hline EQ-5D-5L & $0.20(0.18,0.22)$ & $0.19(0.18,0.21)$ & $0.18(0.16,0.20)$ \\
\hline $\mathrm{BASDAI}^{\mathrm{a}}$ & $-3.3(-3.7,-2.9)$ & $-3.2(-3.6,-2.8)$ & $-2.8(-3.2,-2.4)$ \\
\hline BASDAI $50^{\mathrm{a}, \mathrm{b}}$ & $69.8(61.5,78.2)$ & $68.1(59.7,76.4)$ & $56.1(46.7,65.5)$ \\
\hline $\begin{array}{l}\text { Morning } \\
\text { stiffness }^{\mathrm{a}, \mathrm{c}}\end{array}$ & $-3.2(-3.4,-3.0)^{\dagger}$ & $-3.3(-3.5,-3.0)^{\dagger}$ & $-2.8(-3.0,-2.6)$ \\
\hline SAPS $^{\mathrm{d}}$ & $-29.6(-31.4,-27.9)^{\dagger}$ & $-30.4(-32.2,-28.7)^{*}$ & $-25.8(-27.6,-24.1)$ \\
\hline $\operatorname{Itch}^{\mathrm{d}, \mathrm{e}}$ & $-3.1(-3.3,-2.9)^{\dagger}$ & $-3.2(-3.4,-3.0)^{+}$ & $-2.7(-2.9,-2.5)$ \\
\hline WPAI AI & $-28.2(-30.5,-25.9)^{\dagger}$ & $-26.8(-29.1,-24.4)^{*}$ & $-23.2(-25.5,-20.9)$ \\
\hline $\begin{array}{l}\text { WPAI } \\
\text { presenteeism }^{\mathrm{f}}\end{array}$ & $-25.5(-28.4,-22.7)^{*}$ & $-24.0(-26.8,-21.1)$ & $-20.8(-23.7,-17.9)$ \\
\hline WPAI OWI ${ }^{\mathrm{f}}$ & $-24.5(-28.1,-20.9)$ & $-22.9(-26.5,-19.3)$ & $-20.8(-24.5,-17.1)$ \\
\hline
\end{tabular}


Table 3 continued

\begin{tabular}{llll}
\hline & $\begin{array}{l}\text { Upadacitinib 15 mg QD } \\
(\boldsymbol{N}=371)\end{array}$ & $\begin{array}{l}\text { Upadacitinib 30 mg QD } \\
(\boldsymbol{N}=362)\end{array}$ & $\begin{array}{l}\text { Adalimumab 40 mg EOW } \\
(\boldsymbol{N}=364)\end{array}$ \\
\hline $\begin{array}{l}\text { WPAI } \\
\text { absenteeism }^{\mathrm{f}}\end{array}$ & $-3.0(-5.8,-0.1)$ & $-2.7(-5.5,0.2)$ & $-4.9(-7.8,-2.0)$ \\
\hline
\end{tabular}

$A I$ activity impairment, $B A S D A I$ Bath Ankylosing Spondylitis Disease Activity Index, $B P$ bodily pain, $C I$ confidence interval, $E O W$ every other week, EQ-5D-5L EuroQoL 5-Dimension 5-Level index score, FACIT-F Functional Assessment of Chronic Illness Therapy-Fatigue, GH general health, HAQ-DI Health Assessment Questionnaire Disability Index, $L S$ least squares, $M C S$ Mental Component Summary, $M H$ mental health, $M M R M$ mixed-effects model repeated measurement, NRS numerical rating scale, OWI overall work impairment, $P C S$ Physical Component Summary, $P F$ physical functioning, $P R O$ patient-reported outcome, PtGA Patient Global Assessment of Disease Activity, $Q D$ once daily, $R E$ role emotional, $R P$ role physical, SAPS Self-Assessment of Psoriasis Symptoms, $S F$ social functioning, SF-36 36-Item Short Form Health Survey, $V T$ vitality, $W P A I$ Work Productivity and Activity Impairment

${ }^{*} p<0.05,{ }^{\dagger} P \leq 0.01$, and ${ }^{\ddagger} p \leq 0.001$ for upadacitinib versus adalimumab

a Reported only for patients with investigator-determined psoriatic spondylitis at baseline

b Presented as response rate (95\% CI). NRI. N: upadacitinib $15 \mathrm{mg}$ QD: 116; upadacitinib $30 \mathrm{mg}$ QD: 119; and adalimumab $40 \mathrm{mg}, 107$

${ }^{c}$ Mean of BASDAI questions 5 and 6

d Assessed at week 16 instead of 12

e SAPS question 2

${ }^{\mathrm{f}}$ Reported only for patients who were employed

onset of effect in both pain and function. Significant sustained improvements with upadacitinib treatment in SF-36 PCS, MCS, all eight domains and FACIT-F scores at 24 and 56 weeks indicate a substantial positive impact on HRQoL. This is of particular importance because PsA negatively impacts many aspects of life including patients' psychological and psychosocial well-being, ability to perform daily activities, participation in social activities, as well as the physical and emotional aspects of life [33-37]. At 12 weeks, significantly more patients treated with both doses of upadacitinib reported PtGA, HAQ-DI, FACIT-F, EQ-5D-5L, SF36 PCS, and 6-7 domain scores $\geq$ normative values, indicative of the healthy general population compared with placebo, despite $\leq 11 \%$ having such scores at baseline. These improvements continued to week 56 with the majority reporting scores as if they did not have an inflammatory arthritis such as PsA.

Sustained improvements in WPAI domains with upadacitinib treatment compared with placebo were also reported, and are noteworthy because approximately one-third of respondents in a multinational survey [38] reported that they missed work because of PsA and that their PsA impacted their ability to work full time.

At week 12, improvements in HAQ-DI, SAPS, and SF-36 PCS and PF domain scores with upadacitinib (15 mg: PF or $30 \mathrm{mg}$ : PF, RP, BP and $\mathrm{GH})$ were statistically greater than with adalimumab $40 \mathrm{mg}$ and continued through week 56. Through week 24 , NNTs were $\leq 10$ across most PROs with both doses of upadacitinib and the range of NNTs generally lower than with adalimumab $40 \mathrm{mg}$.

At week 12, reported improvements in PROs were generally numerically greater with upadacitinib $30 \mathrm{mg}$ compared with $15 \mathrm{mg}$. By week 24; however, improvements with both doses of upadacitinib were similar and sustained to week 56. It is important to contextualize the findings of this RCT with other JAK inhibitors indicated for the treatment of PsA such as tofacitinib. In the OPAL Broaden trial [39], significant improvements in PROs at week 12 were reported with tofacitinib (5 or $10 \mathrm{mg}$ twice daily) treatment in DMARD-IR PsA patients. Three of eight SF-36 domain scores [PF, BP, and vitality (VT)] were significantly improved with 


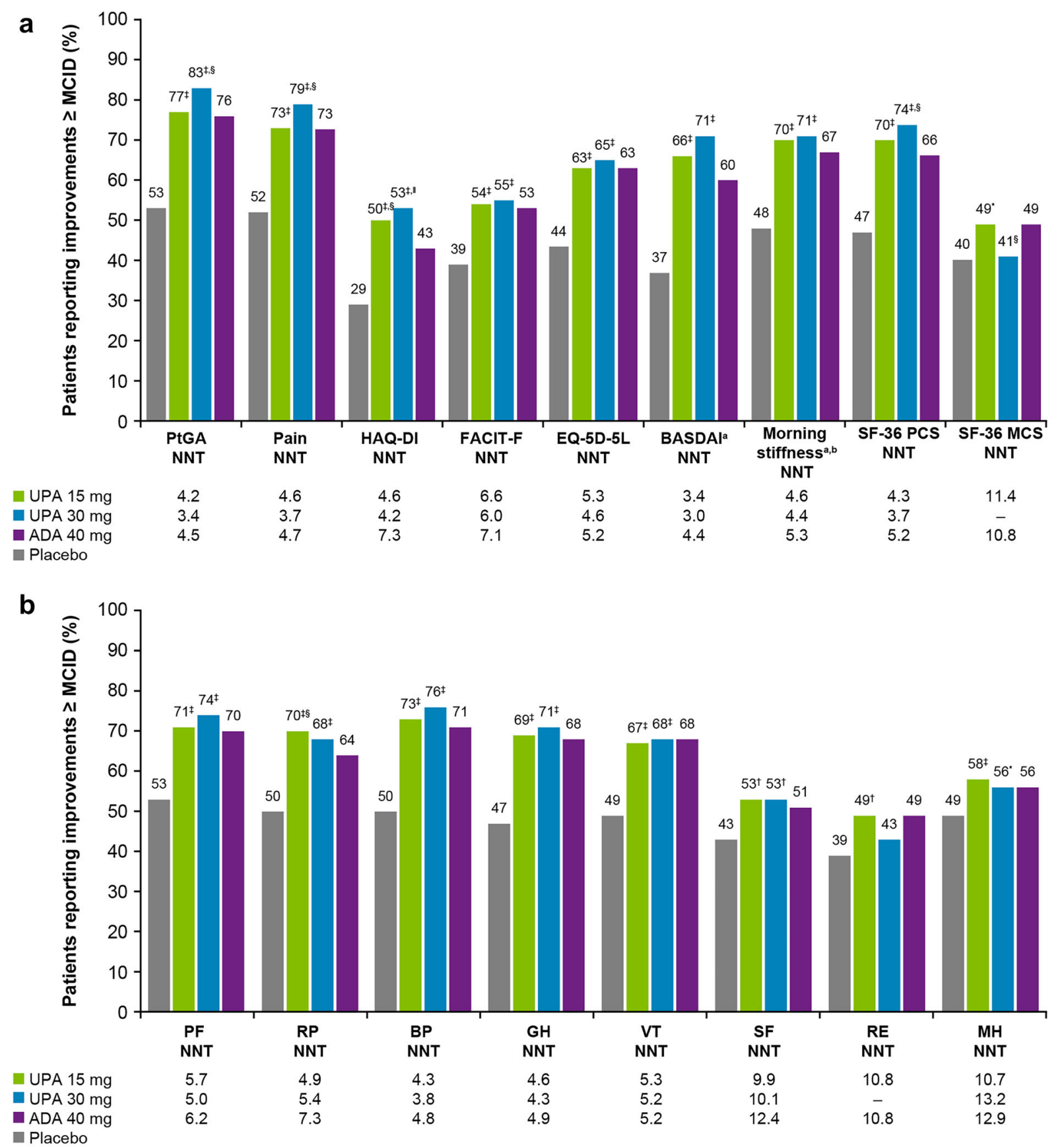


4Fig. 3 Proportion of patients reporting improvements $\geq$ MCID and NNTs in PROs at Week 12 (NRI). a PROs, b SF-36 domains. ${ }^{*} p<0.05,{ }^{\dagger} p \leq 0.01$, and ${ }_{p} \leq 0.001$ UPA versus placebo and ${ }^{s} p<0.05$ and $\|_{p} \leq 0.01$ for UPA versus ADA. aReported only for patients with investigator-determined psoriatic spondylitis at baseline. bMean of BASDAI questions 5 and 6. NNTs were calculated for UPA versus $\mathrm{PBO}$ and for ADA versus PBO. NNT for UPA $30 \mathrm{mg}$ was not calculated for MCS and RE because the proportion of patients reporting improvement was not significantly different for UPA $30 \mathrm{mg}$ versus $\mathrm{PBO}$. MCID definitions: $\geq 1$-point decrease (PtGA, pain, and morning stiffness), $\geq 0.35$-unit decrease (HAQ-DI), $\geq 4$-point increase (FACIT-F), $\geq 0.05$-unit increase (EQ-5D-5L), $\geq 1$ 1.1-point decrease (BASDAI), $\geq 2.5$-point increase (SF-36 PCS and MCS), and $\geq 5$ point increase (SF-36 domains). $A D A$ adalimumab, $B A S D A I$ Bath Ankylosing Spondylitis Disease Activity Index, $B P$ bodily pain, $E Q-5 D-5 L$ EuroQoL 5 -Dimension 5-Level index score, FACIT-F Functional Assessment of Chronic Illness Therapy-Fatigue, $G H$ general health, $H A Q-D I$ Health Assessment Questionnaire Disability Index, $M C I D$ minimal clinically important difference, MCS Mental Component Summary, $M H$ mental health, $N N T$ number needed to treat, $N R I$ non-responder imputation, $P B O$ placebo, $P C S$ Physical Component Summary, $P F$ physical functioning, $P R O$ patient-reported outcome, PtGA Patient Global Assessment of Disease Activity, $R E$ role emotional, $R P$ role physical, $S F$ social functioning, $S F-36$ 36-Item Short Form Health Survey, $U P A$ upadacitinib, $V T$ vitality

both doses of tofacitinib and additionally, the social functioning domain with $5 \mathrm{mg}$ versus placebo at week 12 and were similar to those with adalimumab versus placebo in $\mathrm{PF}, \mathrm{BP}$, and GH domains. In contrast, improvements in HAQ-DI, SAPS, and SF-36 PCS scores following 12 weeks of upadacitinib treatment were greater than adalimumab. Similarly, all eight SF-36 domain scores were significantly improved with upadacitinib treatment versus placebo and exceeded those reported with adalimumab in one (15 mg) and four (30 mg) domains at week 12 in SELECT-PsA 1. Future comparative studies are needed to elucidate the comparative efficacy of JAK inhibitors in PsA patients.

The following limitations should be kept in mind when interpreting the results of this trial. The RCT was not powered to detect differences between the upadacitinib groups. There was no placebo group available for comparison after week 24 . Finally, results may not be generalizable beyond the trial patient population.

\section{CONCLUSIONS}

Treatment with upadacitinib 15 or $30 \mathrm{mg}$ resulted in clinically meaningful improvements in PROs compared with placebo at 12 weeks in non-bDMARD-IR patients with active PsA which were maintained or further improved at weeks 24 and 56. Clinically meaningful improvements in PtGA, pain, and HAQ-DI were evident as early as week 2 with upadacitinib (15 and $30 \mathrm{mg}$ ) and adalimumab $40 \mathrm{mg}$. Overall, improvements were similar between upadacitinib $15 \mathrm{mg}$ QD and adalimumab $40 \mathrm{mg}$ EOW with numerically better results for upadacitinib 

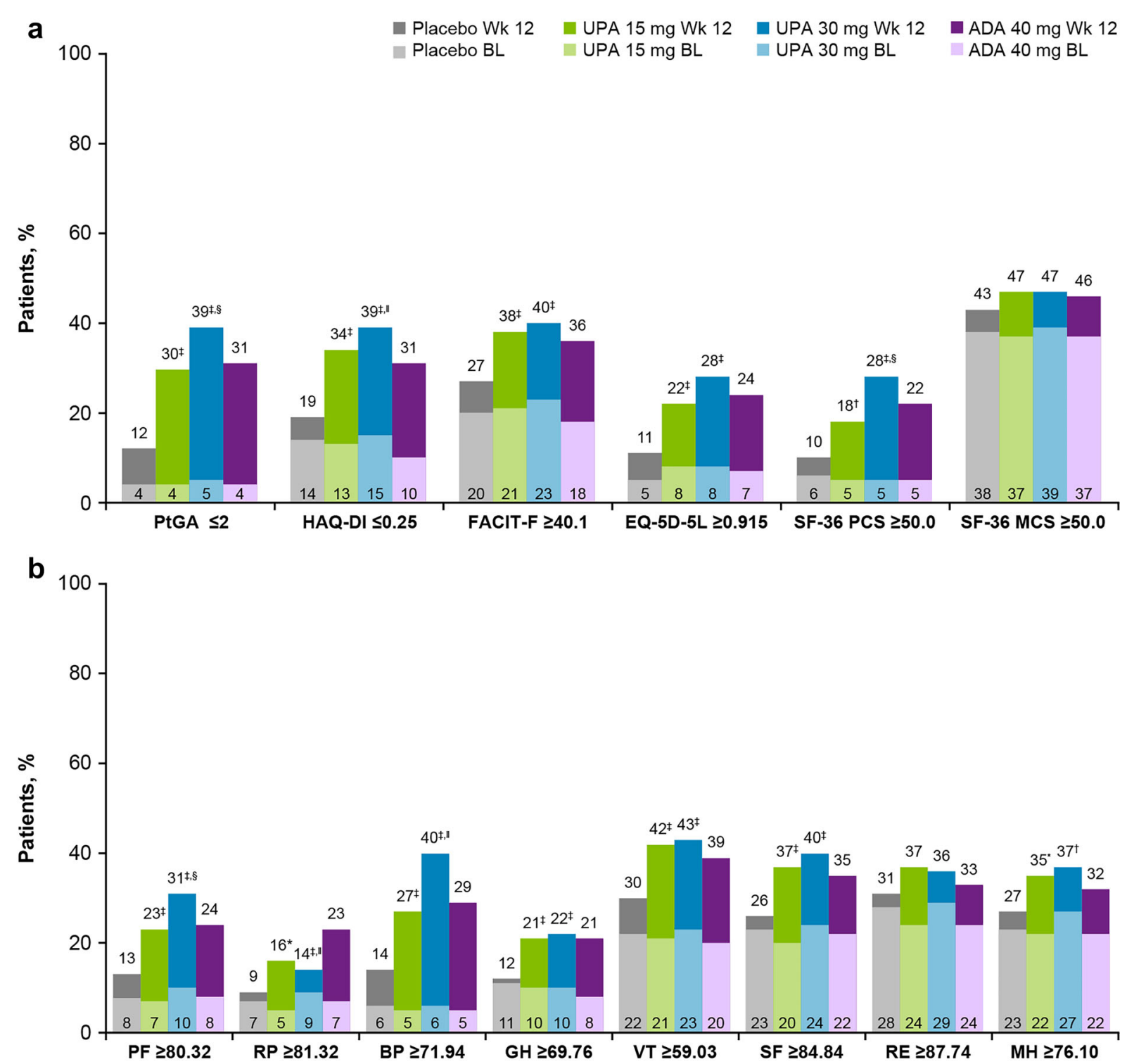

Fig. 4 Proportion of patients reporting PRO scores $\geq$ normative values at baseline and Week 12 (NRI) and age- and gender-matched normative values in SF-36 domains. a PROs, b SF-36 domains. ${ }^{*} p<0.05,{ }^{\dagger} p \leq 0.01$, and ${ }^{\star} p \leq 0.001$ UPA versus PBO and ${ }^{s_{p}}<0.05$ and $\|_{p} \leq 0.01$ for UPA versus ADA. The percentages at 12 weeks may or may not include the same patients that achieved that outcome at baseline. $A D A$ adalimumab, $B L$ baseline, $B P$ bodily pain, EQ-5D-5L EuroQoL 5-Dimension 5-Level index score, FACIT-F Functional Assessment of

30 mg QD in many PROs. Overall, findings from this study demonstrate the potential of upadacitinib treatment to provide significant and clinically meaningful improvements in
Chronic Illness Therapy-Fatigue, $G H$ general health, $H A Q$ DI Health Assessment Questionnaire Disability Index, $M C I D$ minimal clinically important difference, $M C S$ Mental Component Summary, $M H$ mental health, NRI non-responder imputation, $P B O$ placebo, $P C S$ Physical Component Summary, $P F$ physical functioning, $P R O$ patient-reported outcome, $P t G A$ Patient Global Assessment of Disease Activity, $R E$ role emotional, $R P$ role physical, $S F$ social functioning, $S F-36$ 36-Item Short Form Health Survey, $U P A$ upadacitinib, $V T$ vitality

HRQoL and other outcomes most important to patients with PsA. 


\section{ACKNOWLEDGEMENTS}

Funding. Financial support for this study and the journal's Rapid Service Fee and Open Access charge were provided by AbbVie. AbbVie participated in the interpretation of data, review, and approval of the manuscript. All authors contributed to the development of the manuscript and maintained control over the final content. No honoraria or payments were made for authorship.

Authorship. All named authors meet the International Committee of Medical Journal Editors (ICMJE) criteria for authorship for this article, take responsibility for the integrity of the work as a whole, and have given their approval for this version to be published.

Author Contributions. VS contributed to the study concept and design; analysis and interpretation of data; critical revision of the manuscript for important intellectual content; statistical analysis; administration, technical and material support; and study supervision. PJM, ERS, MK, CS, EM-B, KK, and DDG contributed to the study concept and design, analysis, and interpretation of data, and critical revision of the manuscript for important intellectual content. CDS and PZ contributed to study concept and design; acquisition of data; analysis and interpretation of data; critical revision of the manuscript for important intellectual content; statistical analysis; obtained funding, administration, technical and material support; and study supervision.

Medical Writing, Editorial, and Other Assistance. Medical writing assistance was provided by Joann Hettasch, of Fishawack Facilitate Ltd., part of Fishawack Health, and was funded by AbbVie Inc., North Chicago, IL.

Disclosures. V Strand is a consultant for AbbVie, Amgen, Arena, AstraZeneca, Bayer, BMS, Boehringer Ingelheim, Celltrion, Genentech/Roche, GSK, Inmedix, Janssen, Lilly, Merck, Novartis, Pfizer, Regeneron, Samsung, Sandoz, Sanofi, Setpoint, and UCB; and involved in advisory boards for AbbVie, Amgen, Arena, AstraZeneca, BMS, Boehringer Ingelheim, Genentech, GSK, Janssen, Lilly, Merck, Novartis, Pfizer, Regeneron, Samsung, Sanofi, and UCB. PJ Mease received research grants from AbbVie, Amgen, BMS, Celgene, Janssen, Lilly, Novartis, Pfizer, Sun, and UCB; received consulting fees from AbbVie, Amgen, BMS, Celgene, Janssen, Lilly, Novartis, Pfizer, Sun, and UCB; and served on speaker's bureaus for AbbVie, Amgen, BMS, Celgene, Genentech, Janssen, Novartis, Pfizer, and UCB. ER Soriano has received research grants, consulting fees, and/or speaker's fees from AbbVie, Amgen, Bristol Myers Squibb, Celgene, Janssen, Lilly, Novartis, Pfizer, Roche, Sanofi, and UCB. $\mathrm{M}$ Kishimoto has received consulting fees and/ or honoraria from AbbVie, Amgen-Astellas BioPharma, Asahi Kasei Pharma, Astellas, Ayumi Pharma, Boehringer Ingelheim, Bristol Myers Squibb, Celltrion Healthcare, Chugai, DaiichiSankyo, Eisai, Eli Lilly, Gilead, Janssen, Kyowa Kirin, Novartis, Ono, Pfizer, Sanofi, Mitsubishi Tanabe, Teijin Pharma, and UCB. C Salvarani has received consulting fees and research grants from Roche, Sanofi-Genzyme, AbbVie, Pfizer, Lilly, Novartis, and Amgen. K Kato is an AbbVie employee and may own AbbVie stock or options. E McDearmon-Blondell is an AbbVie employee and may own AbbVie stock or options. P Zueger is an AbbVie employee and may own AbbVie stock or options. CD Saffore is an AbbVie employee and may own AbbVie stock or options. DD Gladman has received grant support and/or consulting fees from AbbVie, Amgen, BMS, Eli Lilly, Gilead, Galapagos, Janssen, Novartis, Pfizer, and UCB.

Compliance with Ethics Guidelines. The SELECT-PSA 1 study was conducted according to the International Conference on Harmonization guidelines and the principles of the Declaration of Helsinki. All patients provided written informed consent. The trial protocol was approved by independent ethics committees and institutional review boards (ESM Table S1).

Data Availability. The datasets generated and/or analyzed during the current study are 
available from the corresponding author on reasonable request.

Prior Presentation. Portions of the work were presented at the American College of Rheumatology all-virtual annual meeting, November 5-9, 2020.

Open Access. This article is licensed under a Creative Commons Attribution-NonCommercial 4.0 International License, which permits any non-commercial use, sharing, adaptation, distribution and reproduction in any medium or format, as long as you give appropriate credit to the original author(s) and the source, provide a link to the Creative Commons licence, and indicate if changes were made. The images or other third party material in this article are included in the article's Creative Commons licence, unless indicated otherwise in a credit line to the material. If material is not included in the article's Creative Commons licence and your intended use is not permitted by statutory regulation or exceeds the permitted use, you will need to obtain permission directly from the copyright holder. To view a copy of this licence, visit http://creativecommons.org/licenses/bync/4.0/.

\section{REFERENCES}

1. McGagh D, Coates LC. Assessment of the many faces of PsA: single and composite measures in PsA clinical trials. Rheumatology. 2020;59:i29-36.

2. Husni ME, Merola JF, Davin S. The psychosocial burden of psoriatic arthritis. Semin Arthritis Rheum. 2017;47:351-60.

3. Merola JF, Shrom D, Eaton J, et al. Patient perspective on the burden of skin and joint symptoms of psoriatic arthritis: results of a multi-national patient survey. Rheumatol Ther. 2019;6:33-45.

4. Gossec L, Baraliakos X, Kerschbaumer A, et al. EULAR recommendations for the management of psoriatic arthritis with pharmacological therapies: 2019 update. Ann Rheum Dis. 2020;79:700-12.

5. Ogdie A, Coates LC, Gladman DD. Treatment guidelines in psoriatic arthritis. Rheumatology (Oxford). 2020;59:i37-46.
6. Singh JA, Guyatt G, Ogdie A, et al. Special article: 2018 American College of Rheumatology/National Psoriasis Foundation guideline for the treatment of psoriatic arthritis. Arthritis Rheumatol. 2019;71: 5-32.

7. Parmentier JM, Voss J, Graff C, et al. In vitro and in vivo characterization of the JAK1 selectivity of upadacitinib (ABT-494). BMC Rheumatol. 2018;2: 23.

8. Burmester GR, Kremer JM, Van den Bosch F, et al. Safety and efficacy of upadacitinib in patients with rheumatoid arthritis and inadequate response to conventional synthetic disease-modifying antirheumatic drugs (SELECT-NEXT): a randomised, double-blind, placebo-controlled phase 3 trial. Lancet. 2018;391:2503-12.

9. Fleischmann R, Pangan AL, Song $\mathrm{IH}$, et al. Upadacitinib versus placebo or adalimumab in patients with rheumatoid arthritis and an inadequate response to methotrexate: results of a phase III, double-blind, randomized controlled trial. Arthritis Rheumatol. 2019;71:1788-800.

10. Genovese MC, Fleischmann R, Combe B, et al. Safety and efficacy of upadacitinib in patients with active rheumatoid arthritis refractory to biologic disease-modifying anti-rheumatic drugs (SELECTBEYOND): a double-blind, randomised controlled phase 3 trial. Lancet. 2018;391:2513-24.

11. Smolen JS, Pangan AL, Emery P, et al. Upadacitinib as monotherapy in patients with active rheumatoid arthritis and inadequate response to methotrexate (SELECT-MONOTHERAPY): a randomised, placebocontrolled, double-blind phase 3 study. Lancet. 2019;393:2303-11.

12. van Vollenhoven $R$, Takeuchi $T$, Pangan AL, et al. Efficacy and safety of upadacitinib monotherapy in methotrexate-naïve patients with moderately to severely active rheumatoid arthritis (SELECTEARLY): a randomized, double-blind, active-comparator, multi-center, multi-country trial. Arthritis Rheumatol. 2020;72:1607-20.

13. Smolen JS, Landewé RBM, Bijlsma JWJ, et al. EULAR recommendations for the management of rheumatoid arthritis with synthetic and biological disease-modifying antirheumatic drugs: 2019 update. Ann Rheum Dis. 2020;79:685-99.

14. McInnes IB, Anderson JK, Magrey M, et al. Trial of upadacitinib and adalimumab for psoriatic arthritis. N Engl J Med. 2021;384:1227-39.

15. Ogdie A, de Wit M, Callis Duffin K, et al. Defining outcome measures for psoriatic arthritis: a report from the GRAPPA-OMERACT working group. J Rheumatol. 2017;44:697-700. 
16. Taylor W, Gladman D, Helliwell P, et al. Classification criteria for psoriatic arthritis: development of new criteria from a large international study. Arthritis Rheum. 2006;54:2665-73.

17. Mease PJ, Woolley JM, Bitman B, et al. Minimally important difference of Health Assessment Questionnaire in psoriatic arthritis: relating thresholds of improvement in functional ability to patientrated importance and satisfaction. J Rheumatol. 2011;38:2461-5.

18. Krishnan E, Sokka T, Häkkinen A, et al. Normative values for the Health Assessment Questionnaire disability index: benchmarking disability in the general population. Arthritis Rheum. 2004;50: 953-60.

19. Hewlett S, Dures E, Almeida C. Measures of fatigue: Bristol Rheumatoid Arthritis Fatigue Multi-Dimensional Questionnaire (BRAF MDQ), Bristol Rheumatoid Arthritis Fatigue Numerical Rating Scales (BRAF NRS) for severity, effect, and coping, Chalder Fatigue Questionnaire (CFQ), Checklist Individual Strength (CIS20R and CIS8R), Fatigue Severity Scale (FSS), Functional Assessment Chronic Illness Therapy (Fatigue) (FACIT-F), Multi-Dimensional Assessment of Fatigue (MAF), Multi-Dimensional Fatigue Inventory (MFI), Pediatric Quality Of Life (PedsQL) Multi-Dimensional Fatigue Scale, Profile of Fatigue (ProF), Short Form 36 Vitality Subscale (SF-36 VT), and Visual Analog Scales (VAS). Arthritis Care Res (Hoboken). 2011;63(Suppl 11): S263-286.

20. Webster K, Cella D, Yost K. The Functional Assessment of Chronic Illness Therapy (FACIT) measurement system: properties, applications, and interpretation. Health Qual Life Outcomes. 2003;1: 79.

21. Ware J, Kosinski M, Bjorner J, et al editors. User's Manual for the SF-36v2 ${ }^{\circledR}$ Health Survey. Lincoln: Quality Metric, Inc.; 2007.

22. Ware JE Jr, Kosinski M, Bayliss MS, et al. Comparison of methods for the scoring and statistical analysis of SF-36 health profile and summary measures: summary of results from the Medical Outcomes Study. Med Care. 1995;33:AS264-79.

23. Strand V, Boers M, Idzerda L, et al. It's good to feel better but it's better to feel good and even better to feel good as soon as possible for as long as possible. Response criteria and the importance of change at OMERACT 10. J Rheumatol. 2011;38:1720-7.

24. Armstrong AW, Banderas B, Foley C, et al. Development and psychometric evaluation of the selfassessment of psoriasis symptoms (SAPS)—clinical trial and the SAPS-real world patient-reported outcomes. J Dermatolog Treat. 2017;28:505-14.
25. Salaffi F, Stancati A, Silvestri CA, et al. Minimal clinically important changes in chronic musculoskeletal pain intensity measured on a numerical rating scale. Eur J Pain. 2004;8:283-91.

26. Anderson JK, Zimmerman L, Caplan L, et al. Measures of rheumatoid arthritis disease activity: Patient (PtGA) and Provider (PrGA) Global Assessment of Disease Activity, Disease Activity Score (DAS) and Disease Activity Score with 28-Joint Counts (DAS28), Simplified Disease Activity Index (SDAI), Clinical Disease Activity Index (CDAI), Patient Activity Score (PAS) and Patient Activity Score-II (PASII), Routine Assessment of Patient Index Data (RAPID), Rheumatoid Arthritis Disease Activity Index (RADAI) and Rheumatoid Arthritis Disease Activity Index-5 (RADAI-5), Chronic Arthritis Systemic Index (CASI), Patient-Based Disease Activity Score With ESR (PDAS1) and PatientBased Disease Activity Score without ESR (PDAS2), and Mean Overall Index for Rheumatoid Arthritis (MOI-RA). Arthritis Care Res (Hoboken). 2011;63(Suppl 11):S14-36.

27. Dolan P. Modeling valuations for EuroQol health states. Med Care. 1997;35:1095-108.

28. Hinz A, Kohlmann T, Stöbel-Richter Y, et al. The quality of life questionnaire EQ-5D-5L: psychometric properties and normative values for the general German population. Qual Life Res. 2014;23:443-7.

29. Kviatkovsky MJ, Ramiro S, Landewé R, et al. The minimum clinically important improvement and patient-acceptable symptom state in the BASDAI and BASFI for patients with ankylosing spondylitis. J Rheumatol. 2016;43:1680-6.

30. Reilly Associates. Work productivity and activity questionnaire specific health problem V2.0 (WPAISHP). 2010. http://www.reillyassociates.net/WPAI_ SHP.html. Accessed 2 Feb 2021.

31. Reilly MC, Zbrozek AS, Dukes EM. The validity and reproducibility of a work productivity and activity impairment instrument. Pharmacoeconomics. 1993;4:353-65.

32. Strand V, Crawford B, Singh J, et al. Use of "spydergrams" to present and interpret SF-36 healthrelated quality of life data across rheumatic diseases. Ann Rheum Dis. 2009;68:1800-4.

33. Lee S, Mendelsohn A, Sarnes E. The burden of psoriatic arthritis: a literature review from a global health systems perspective. P T. 2010;35:680-9.

34. Strand V, Sharp V, Koenig AS, et al. Comparison of health-related quality of life in rheumatoid arthritis, psoriatic arthritis and psoriasis and effects of etanercept treatment. Ann Rheum Dis. 2012;71: 1143-50. 
35. Salaffi F, Carotti M, Gasparini S, et al. The healthrelated quality of life in rheumatoid arthritis, ankylosing spondylitis, and psoriatic arthritis: a comparison with a selected sample of healthy people. Health Qual Life Outcomes. 2009;7:25.

36. Kavanaugh A, Gottlieb A, Morita A, et al. The contribution of joint and skin improvements to the health-related quality of life of patients with psoriatic arthritis: a post hoc analysis of two randomised controlled studies. Ann Rheum Dis. 2019;78: 1215-9.

37. Gudu T, Gossec L. Quality of life in psoriatic arthritis. Expert Rev Clin Immunol. 2018;14: 405-17.
38. Kavanaugh A, Helliwell P, Ritchlin CT. Psoriatic arthritis and burden of disease: patient perspectives from the population-based multinational assessment of psoriasis and psoriatic arthritis (MAPP) survey. Rheumatol Ther. 2016;3:91-102.

39. Strand V, de Vlam K, Covarrubias-Cobos JA, et al. Tofacitinib or adalimumab versus placebo: patientreported outcomes from OPAL Broaden-a phase III study of active psoriatic arthritis in patients with an inadequate response to conventional synthetic disease-modifying antirheumatic drugs. RMD Open. 2019;5:e000806. 